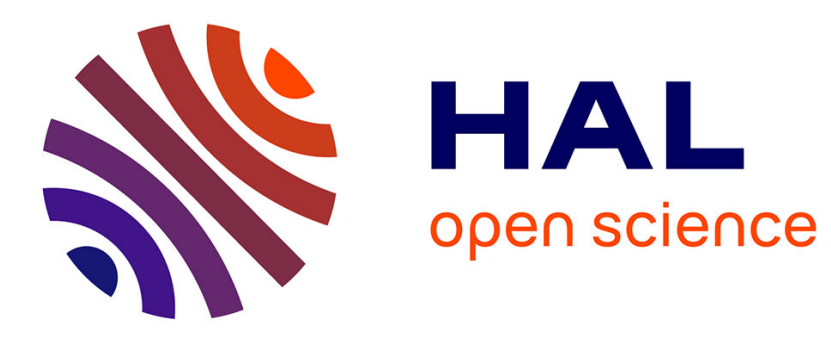

\title{
L'importance des exclus de l'intégration monétaire en Europe
}

Philippe Martin

\section{To cite this version:}

Philippe Martin. L'importance des exclus de l'intégration monétaire en Europe. Revue Economique, 1996, 47 (3), pp.807-817. 10.3406/reco.1996.409819 . hal-01010094

\section{HAL Id: hal-01010094 \\ https://hal-sciencespo.archives-ouvertes.fr/hal-01010094}

Submitted on 19 Jun 2014

HAL is a multi-disciplinary open access archive for the deposit and dissemination of scientific research documents, whether they are published or not. The documents may come from teaching and research institutions in France or abroad, or from public or private research centers.
L'archive ouverte pluridisciplinaire HAL, est destinée au dépôt et à la diffusion de documents scientifiques de niveau recherche, publiés ou non, émanant des établissements d'enseignement et de recherche français ou étrangers, des laboratoires publics ou privés.

\section{(이)(\$)}

Distributed under a Creative Commons Attribution - NonCommercial - NoDerivatives| 4.0 


\title{
Persée
}

\author{
http://www.persee.fr
}

\section{L'importance des exclus de l'intégration monétaire en Europe.}

\author{
Philippe Martin \\ Revue économique, Année 1996, Volume 47, Numéro 3 \\ p. 807 - 817
}

Voir l'article en ligne

\section{Avertissement}

L'éditeur du site " PERSEE » - le Ministère de la jeunesse, de l'éducation nationale et de la recherche, Direction de l'enseignement supérieur, Sous-direction des bibliothèques et de la documentation - détient la propriété intellectuelle et les droits d'exploitation. A ce titre il est titulaire des droits d'auteur et du droit sui generis du producteur de bases de données sur ce site conformément à la loi n`98-536 du 1 er juillet 1998 relative aux bases de données.

Les oeuvres reproduites sur le site «PERSEE » sont protégées par les dispositions générales du Code de la propriété intellectuelle.

Droits et devoirs des utilisateurs

Pour un usage strictement privé, la simple reproduction du contenu de ce site est libre.

Pour un usage scientifique ou pédagogique, à des fins de recherches, d'enseignement ou de communication excluant toute exploitation commerciale, la reproduction et la communication au public du contenu de ce site sont autorisées, sous réserve que celles-ci servent d'illustration, ne soient pas substantielles et ne soient pas expressément limitées (plans ou photographies). La mention Le Ministère de la jeunesse, de l'éducation nationale et de la recherche, Direction de l'enseignement supérieur, Sous-direction des bibliothèques et de la documentation sur chaque reproduction tirée du site est obligatoire ainsi que le nom de la revue et- lorsqu'ils sont indiqués - le nom de l'auteur et la référence du document reproduit.

Toute autre reproduction ou communication au public, intégrale ou substantielle du contenu de ce site, par quelque procédé que ce soit, de l'éditeur original de l'oeuvre, de l'auteur et de ses ayants droit.

La reproduction et l'exploitation des photographies et des plans, y compris à des fins commerciales, doivent être autorisés par l'éditeur du site, Le Ministère de la jeunesse, de l'éducation nationale et de la recherche, Direction de l'enseignement supérieur, Sous-direction des bibliothèques et de la documentation (voir http://www.sup.adc.education.fr/bib/ ). La source et les crédits devront toujours être mentionnés. 


\title{
L'importance des exclus de l'intégration monétaire en Europe
}

\author{
Philippe Martin*
}

\begin{abstract}
Contrairement à l'analyse traditionnelle, nous analysons les coûts associés non pas à l'inclusion mais à l'exclusion d'un pays de l'Union économique et monétaire européenne (UEM). Dans un modèle à trois pays, nous montrons que la politique monétaire du pays exclu sera plus expansionniste et plus contre-cyclique que celle des pays de l'UEM, ce qui la rapproche d'une stratégie de dépréciation compétitive. Le pays dont les chocs sont les plus symétriques à ceux du pays exclu sera le plus touché par cette exclusion voyant la variance de sa production augmenter. Nous examinons aussi les diverses propositions de gestion des relations entre I'UEM et les pays non UEM de l'UE.
\end{abstract}

\section{THE IMPORTANCE OF EXCLUDED COUNTRIES OF MONETARY INTEGRATION IN EUROPE}

In contrast to the traditional analysis, this paper analyses the costs implied by the exclusion rather than by the inclusion of a country to the European Monetary Union (EMU). In a three-country model, we show that the monetary policy of the excluded country will be more expansionist and more counter-cyclical than the monetary policy of the EMU countries. This resembles a strategy of competitive depreciation. The country whose shocks are most symmetric to those of the excluded country suffers most from this exclusion, through an increase in the variance of its production. We also examine the proposals to deal with the relations between EMU and non EMU countries of the EU.

Classification JEL : F33, F42

\section{INTRODUCTION}

La réflexion sur l'intégration monétaire en Europe s'est concentrée sur deux questions. D'une part, les économistes ont utilisé la théorie des zones monétaires optimales pour déterminer si l'Union européenne constituait une telle zone. D'autre part, les praticiens se sont attachés à déterminer les modalités de la transition vers l'Union économique et monétaire européenne (UEM). Dans les deux cas, ces réflexions ont conduit à exclure, au moins de manière temporaire, un certain nombre de pays du processus d'intégration monétaire alors même qu'ils

* Institut des hautes études internationales, 11A, avenue de la Paix, 1202 Genève, Suisse ; CEPI, Paris et CEPR, Londres.

Je remercie Benoît Coeuré, Lionel Fontagné, Jacques Melitz, Jean Pisani-Ferry et Charles Wiplosz pour leurs commentaires. 
sont partie intégrante du Marché unique ${ }^{1}$. Dans le premier cas, le travail empirique de Bayoumi et Eichengreen [1993] suggère que, seuls, quelques pays du cœur de l'Europe (Allemagne, Benelux, Autriche, Danemark, France) partagent des chocs suffisamment symétriques pour qu'une union monétaire entre ces pays n'engendre pas des coûts trop élevés. Dans le second cas, ce sont les critères de convergence de Maastricht, et en particulier ceux liés à l'inflation et à la situation budgétaire, qui ont servi à exclure de fait des pays de l'intégration monétaire. Dans les deux types d'analyse, ce sont les coûts de l'inclusion dans l'UEM qui ont servi à déterminer quels sont les pays qui devraient participer à l'UEM.

Ainsi, le choix implicite de l'exclusion de l'Italie a été fait selon la réflexion, essentiellement d'origine allemande, que l'Italie à l'intérieur impliquerait des coûts trop élevés du fait de la situation de sa dette publique. On se rend compte aujourd'hui, à l'occasion de la dépréciation «compétitive » de la lire, que l'exclusion de l'Italie implique aussi des coûts pour les pays qui ont décidé de demeurer dans le SME et, à terme, de former une union monétaire. On a semblé réfléchir comme si la formation d'une zone de change fixe ou d'une union monétaire en Europe n'avait pas d'impact sur la politique monétaire des pays exclus.

Nous voulons, dans cet article, renverser cette perspective et réfléchir aux implications de la création de l'UEM sur la politique monétaire des pays exclus et l'effet en retour que celle-ci aura sur les pays de l'UEM. Contrairement à l'analyse traditionnelle de la théorie des zones monétaires optimales, notre analyse portera donc sur les coûts associés non pas à l'inclusion mais à l'exclusion d'un pays d'une union monétaire.

La première section expose le modèle théorique que nous allons utiliser. Les deuxième et troisième parties analysent respectivement les politiques monétaires et les effets sur la production des trois pays dans les différents systèmes de change. La dernière partie examine les propositions de gestion des relations entre l'UEM et les devises non UEM de l'UE.

\section{UN MODÈLE À TROIS PAYS}

Le modèle que nous avons utilisé est très proche de celui de Martin [1995] lui-même dérivé du modèle Barro-Gordon [1983]. Il s'agit d'un modèle à trois pays de taille égale que nous appelons la France (F), l'Allemagne (A) et l'Italie (I). La fonction de perte des trois pays dépend du taux d'inflation $\pi$ et de la déviation du log du niveau de production $y$ par rapport au niveau socialement optimal de production $\bar{y}:^{2}$

$$
\mathrm{L}_{i}=\frac{1}{2} \pi_{i}^{2}+\frac{1}{2} \beta\left(y_{i}-\bar{y}_{i}\right)^{2} \quad i=\mathrm{F}, \mathrm{A}, \mathrm{I}
$$

1. Les problèmes posés par une intégration multi-vitesses sont analysés par Alesina et Grilli [1993], Laskar [1995], Pisani-Ferry [1995], Martin [1994b], Eichengreen et Ghironi [1995], Martin [1995], Martin et Ottoviano [1995] et Artus [1995].

2. Par souci de simplification, nous ferons l'hypothèse que $\bar{y}$ est identique en Allemagne et en France. 
où $\beta$ représente le poids relatif placé par l'autorité monétaire sur la stabilisation de la production par rapport à celle de l'inflation. Le jeu étant identique chaque période et excluant les phénomènes de réputation, les politiques optimales sont analysées pour une seule période.

Les trois pays produisent le même bien, la loi du prix unique s'applique et la fonction de production de la France est la suivante :

$$
y_{\mathrm{F}}=\left(p_{\mathrm{F}}-w_{\mathrm{F}}\right)-\frac{1}{2}\left(p_{\mathrm{A}}-w_{\mathrm{A}}\right)-\frac{1}{2}\left(p_{\mathrm{I}}-w_{\mathrm{I}}\right)+\varepsilon
$$

où $w$ est le $\log$ du salaire, $p$ le $\log$ du niveau des prix et $\varepsilon$ un choc de productivité distribué $\mathrm{N}(0, \sigma)$ et que l'on considère commun aux trois pays pour l'instant. Selon les critères de Mundell, ces trois pays constituent donc une zone monétaire optimale. Les fonctions de production allemande et italienne sont symétriques. Du fait de l'existence de contrats, le choc n'est pas observé par les salariés au moment de la détermination des salaires mais il l'est par la Banque centrale lorsque celle-ci choisit sa politique monétaire. Le premier terme de l'équation, le salaire réel, est la partie standard du modèle Barro-Gordon. Les deuxième et troisième termes de l'équation prennent en compte la forte interdépendance qui existe au sein de l'UE : on peut les interpréter en pensant à une firme multinationale qui a des usines dans ces trois pays européens et doit décider où augmenter ou diminuer l'emploi et la production. L'équation (2) implique que cette entreprise choisira d'augmenter la production relativement plus dans le pays au plus faible salaire réel ${ }^{1}$. Cette modélisation permet d'obtenir une courbe de Phillips à pente positive au niveau national et verticale au niveau européen ${ }^{2}$. Cela implique donc qu'il existera une concurrence entre les pays pour attirer la production et l'emploi, celle-ci se faisant au niveau du salaire réel. Si les salariés veulent minimiser les variations de leur salaire réel, leur stratégie optimale est de les augmenter au niveau de l'inflation anticipée $\pi_{i}^{e}$. La fonction de production de la France devient ainsi :

$$
y_{\mathrm{F}}=\left(\pi_{\mathrm{F}}-\pi_{\mathrm{F}}^{e}\right)-\frac{1}{2}\left(\pi_{\mathrm{A}}-\pi_{\mathrm{A}}^{e}\right)-\frac{1}{2}\left(\pi_{\mathrm{I}}-\pi_{\mathrm{I}}^{e}\right)+\varepsilon
$$

\section{LES POLITIQUES MONÉTAIRES OPTIMALES}

Notre premier objectif est de déterminer les politiques monétaires optimales dans les trois équilibres institutionnels qui nous intéressent : taux de change flexible, taux de change fixe en Europe, taux de change fixe entre la France et l'Allemagne excluant l'Italie. Dans notre modèle, l'UEM est identique à une

1. L'exemple de Hoover délocalisant ses activités de Dijon en Écosse, après la sortie de la Grande-Bretagne du SME en 1992, montre qu'un mécanisme semblable à celui décrit ici est à l'œuvre, avec les répercussions politiques, si ce n'est économiques, que l'on connaît.

2. Une pente positive aurait pu être choisie au niveau européen sans changer la nature des résultats. L'hypothèse importante est que, du fait de la compétition entre pays pour attirer la production, la pente est plus forte au niveau européen qu'au niveau national. 
zone de taux de change fixe irrémédiable. Nous interpréterons donc nos résultats à la fois pour la formation de l'UEM et dans une certaine mesure pour un SME « dur».

L'UEM complète est l'équilibre le plus facile à déterminer. Dans ce cas, la politique monétaire et les taux d'inflation sont identiques dans les trois pays, ce qui élimine toute possibilité d'arbitrage entre inflation et production à court terme. La politique monétaire optimale, parfaitement anticipée par les agents, est donc de ne pas réagir aux chocs temporaires : l'inflation est nulle pour toutes les réalisations du choc $\varepsilon$. C'est la représentation théorique extrême d'une union monétaire complète. Prenons maintenant le cas des taux de change flexibles. Chaque pays minimise sa fonction de perte en choisissant son taux d'inflation optimal prenant comme données le taux d'inflation national anticipé et les taux d'inflation étrangers. Il y a donc, de la part de la Banque centrale nationale, un jeu à la fois avec les agents nationaux et avec les banques centrales étrangères. L'équilibre de Nash que l'on détermine implique les taux d'inflation anticipés et les taux d'inflation effectifs suivants :

$$
\pi_{i}^{e}=\beta \bar{y}_{i} ; \pi_{i}=\beta \bar{y}_{i}-\beta \varepsilon \quad i=\mathrm{F}, \mathrm{A}, \mathrm{I}
$$

En change flexible, le taux d'inflation anticipé et moyen est supérieur à celui en change fixe et dépend de la déviation entre le niveau de production naturel et le niveau de production socialement optimal $\tilde{y}$. Ainsi, plus les distorsions internes associées à cette déviation, par exemple le niveau des impôts, sont fortes, plus l'inflation moyenne sera élevée. Les changes flexibles introduisent un biais inflationniste car les banques centrales tentent d'atteindre le niveau de production optimal en diminuant le salaire réel dans leur pays plus que dans les autres afin d'attirer la production et l'emploi. Les salariés anticipent parfaitement cette partie de la politique de dépréciation compétitive et demandent donc des augmentations de salaires conséquentes. En change flexible, une politique monétaire de faible inflation n'est pas crédible vis-à-vis des salariés nationaux. En outre, une politique monétaire de faible réaction aux chocs n'est pas crédible vis-à-vis des banques centrales étrangères. L'inflation est trop variable en système de change flexible car les autorités monétaires réagissent à un choc négatif en diminuant le salaire réel ${ }^{1}$.

Venons-en maintenant au cas le plus intéressant, c'est-à-dire un système de change fixe, ou une union monétaire, entre la France et l'Allemagne, excluant l'Italie $^{2}$. La France et l'Allemagne prennent en compte, lorsqu'elles déterminent leur politique monétaire optimale, le fait que leur taux d'inflation sera identique à l'équilibre. La Banque centrale italienne prend aussi en compte cette contrainte de la politique monétaire franco-allemande. Les fonctions de production de la France (identique à celle de l'Allemagne) et de l'Italie sont désormais :

$$
y_{\mathrm{F}}=\frac{1}{2}\left(\pi_{\mathrm{F}}-\pi_{\mathrm{F}}^{e}\right)-\frac{1}{2}\left(\pi_{\mathrm{I}}-\pi_{\mathrm{I}}^{e}\right)+\varepsilon ; y_{\mathrm{I}}=\left(\pi_{\mathrm{I}}-\pi_{\mathrm{I}}^{e}\right)-\left(\pi_{\mathrm{F}}-\pi_{\mathrm{F}}^{e}\right)+\varepsilon
$$

1. Lors d'une surchauffe de l'économie, la Banque centrale tentera de manière symétrique d'exporter le « surplus » de production et ainsi réduire le taux d'inflation.

2. Nous n'analysons pas ici les raisons pour lesquelles la France et l'Allemagne veulent exclure l'Italie. Celles-ci sont étudiées dans le cadre de ce modèle dans Martin [1995]. 
Les taux d'inflation anticipés et réalisés des différents pays en équilibre de Nash sont donc :

$$
\begin{gathered}
\pi_{i}^{e}=\frac{1}{2} \beta \bar{y}_{i} ; \pi_{i}=\frac{1}{2} \beta \bar{y}_{i}-\beta \frac{2+3 \beta}{4+5 \beta} \varepsilon \quad i=\mathrm{F}, \mathrm{A} \\
\pi_{\mathrm{I}}^{e}=\beta \bar{y}_{\mathrm{I}} ; \pi_{\mathrm{I}}=\beta \bar{y}_{\mathrm{I}}-\beta \frac{4+3 \beta}{4+5 \beta} \varepsilon
\end{gathered}
$$

Pour l'Allemagne et la France, cet équilibre implique une inflation moyenne plus faible et moins variable qu'en change flexible $[(2+3 \beta) /(4+5 \beta)<1]$. Pour l'Italie, l'inflation moyenne est la même que lorsque l'Allemagne et la France étaient en change flexible.

Alors que l'écart d'inflation moyenne augmente entre l'Italie et ses partenaires du fait de la création d'une zone partielle de change fixe, l'inflation italienne devient moins variable même si la politique monétaire italienne réagit d'avantage aux chocs transitoires que celle de ses partenaires $[(2+3 \beta) /(4+5 \beta)<$ $(4+3 \beta) /(4+5 \beta)$ dans l'équation (6)]. Lorsque la France fixe son taux de change avec l'Allemagne, cela implique qu'elle internalise une partie de l'externalité qui existe au niveau européen puisqu'elle ne tente plus d'exporter le choc vers l'Allemagne. Les deux pays ont donc intérêt à réduire leur réaction au choc.

La politique monétaire italienne devient plus active que lorsqu'il existe un système de change fixe entre les trois pays. On peut interpréter ce résultat aussi en termes d'effet de taille des pays (Martin [1994]). En effet, l'Italie bénéficie désormais de sa taille relativement petite vis-à-vis du bloc France-Allemagne. Si ce bloc avait un taux d'inflation non anticipé supérieur à celui de l'Italie, le transfert de production ne se ferait qu'en provenance d'un seul pays et devrait être partagé entre les deux pays. Pour l'Italie, c'est exactement l'inverse. On peut donc interpréter la sortie de l'Italie du SME « dur » dans ce contexte théorique : en sortant du SME, l'Italie non seulement regagne une marge de manœuvre importante en termes de stabilisation de la production mais elle profite aussi de la zone de faible inflation et de politique monétaire moins « active» que constitue toujours le couple franco-allemand. L'Italie peut ainsi utiliser sa politique monétaire pour stabiliser son économie contre un choc négatif étant donné que les membres de l'UEM ont optimalement choisi une politique moins contre-cyclique. Nous pouvons donc interpréter la dépréciation « compétitive » de la lire dans ce contexte théorique de free-riding. Certes, la politique monétaire et de change sont, dans notre modèle, déterminées par la Banque centrale et non pas imposées par les marchés. Il n'est pas certain qu'il y ait, au moins ex-post, une grande différence entre une dépréciation compétitive interprétée comme stratégie délibérée ou comme imposée par le marché. En effet, si les agents sur les marchés ont en tête un modele semblable à celui développé ici, toute autre politique que la politique monétaire optimale déterminée ici ne sera pas crédible aux yeux des marchés.

\section{LA PRODUCTION}

En système de change fixe et flexible où les trois pays ont un statut identique, la politique monétaire n'a pas d'effet sur la production est donc égale au choc $\varepsilon$. En change fixe, c'est parce que la politique monétaire optimale est une inflation constante et nulle. En change flexible, $c$ 'est parce que la politique monétaire est 
identique dans les trois pays. Si les banques centrales parviennent bien à diminuer le salaire réel en réaction au choc puisque celui-ci n'est pas anticipé par les agents lors de la signature des contrats, elles sont en revanche incapables d'influencer le niveau de production puisque chacune des banques centrales, dans cet équilibre, adopte la même stratégie qui s'apparente à une dépréciation compétitive. À l'équilibre, en récession, le salaire réel a été diminué dans les différents pays mais sans bénéfice pour la production.

En revanche, lorsque l'Allemagne et la France sont en change fixe et l'Italie en change flexible, les productions respectives sont :

$$
y_{i}=\frac{4+6 \beta}{4+5 \beta} \varepsilon \quad i=\mathrm{F}, \mathrm{A} \quad ; \quad y_{\mathrm{I}}=\frac{4+3 \beta}{4+5 \beta} \varepsilon
$$

La production en Allemagne et en France devient moins stable: $(4+6 \beta) /(4+5 \beta) \varepsilon>\varepsilon$. Celle de l'Italie devient plus stable puisque désormais l'Italie, exploitant le système de change fixe entre l'Allemagne et la France, a une politique monétaire plus efficace : $(4+3 \beta) /(4+5 \beta) \varepsilon<\varepsilon$. L'exclusion de pays membres à part entière de l'UE du processus de l'intégration monétaire va donc créer des conflits entre les membres de l'UEM et les pays exclus. Pour tous les pays, l'inflation est trop élevée et trop variable du fait de l'exclusion de l'Italie de la zone de change fixe. Notons cependant que l'Allemagne et la France ont toujours intérêt à former une zone de change fixe même partielle car l'augmentation de la variance de la production est plus que compensée dans la fonction de perte par la diminution à la fois de la moyenne et de la variance de l'inflation. Martin [1995] montre que le pays exclu peut, une fois invité, avoir intérêt a conserver cette position de free-rider.

La France et l'Allemagne sont-elles dans des positions différentes vis-à-vis du choix d'exclusion dont nous venons d'analyser les coûts en termes de variance de la production? Jusqu'ici, ces deux pays sont identiques et les effets de l'exclusion de l'Italie sur ces deux pays le sont donc aussi. Supposons maintenant que ces trois pays soient affectés par des chocs stochastiques différents, $\varepsilon_{\mathrm{A}}, \varepsilon_{\mathrm{F}}, \varepsilon_{\mathrm{I}}$. Nous voulons montrer que même si la variance de ces chocs est identique, la variance de la production dans chaque pays dépend de la corrélation des chocs avec ceux du pays exclu. Ainsi, si l'Allemagne et la France creent une zone de change fixe excluant l'Italie, les taux d'inflation optimaux sont ${ }^{1}$ :

$$
\begin{gathered}
\pi_{A}=\pi_{F}=\frac{1}{2} \beta \bar{y}_{F}-\frac{\beta}{4+5 \beta}\left[(1+\beta)\left(\varepsilon_{A}+\varepsilon_{F}\right)+\beta \varepsilon_{I}\right] \\
\pi_{I}=\beta \bar{y}_{1}-\frac{\beta}{4+5 \beta}\left[\beta\left(\varepsilon_{A}+\varepsilon_{F}\right)+(4+\beta) \varepsilon_{I}\right]
\end{gathered}
$$

On peut montrer aisément que lorsque la variance des chocs est égale dans les trois pays à $\sigma^{2}$, la différence de la variance de la production en France et en Allemagne est la suivante :

$$
\operatorname{var}\left(y_{\mathrm{F}}\right)-\operatorname{var}\left(y_{\mathrm{A}}\right)=\frac{2 \beta \sigma^{2}}{4+5 \beta}[\rho(\mathrm{F}, \mathrm{I})-\rho(\mathrm{A}, \mathrm{I})]
$$

1. Pour calculer ces stratégies optimales, nous faisons l'hypothèse standard qu'un banquier central européen minimise la somme des fonctions de perte de l'Allemagne et de la France. 
où $\rho(\mathrm{F}, \mathrm{I})$ et $\rho(\mathrm{A}, \mathrm{I})$ sont respectivement les coefficients de corrélation des chocs français et italiens d'une part et des chocs allemands et italiens d'autre part. Ainsi, si les chocs français sont plus corrélés à ceux de l'Italie que ne le sont les chocs allemands, comme le suggèrent les travaux du CEPR [1991] et de Bayoumi et Eichengreen [1993], c'est-à-dire que $\rho(F, I)>\rho(A, I)$, la France subira plus fortement les conséquences de la politique monétaire d'une Italie exclue. En effet, pendant une récession forte à la fois en Italie et en France, mais moindre en Allemagne, l'Italie conduira une politique de dépréciation permettant d'importer de la production de la zone franco-allemande. Du fait de la différence de corrélation des chocs, la perte de production est subie en France au moment de la récession, alors qu'en Allemagne elle se fait dans une autre phase du cycle. De ce fait, la variance de la production sera plus forte en France qu'en Allemagne. Une autre conséquence de ce résultat est que l'exclusion d'un pays du processus de l'intégration monétaire générera des conflits d'intérêt entre les pays qui font partie de l'UEM. Dans ce cas, en effet, la réaction optimale de la Banque centrale européenne à la politique monétaire des pays exclus ne sera pas la même pour les différents membres de l'UEM.

Selon ces résultats, les critères de choix d'inclusion ou d'exclusion des pays d'une union monétaire sont proches de ceux énoncés dans la théorie des zones monétaires optimales de Mundell [1961]. Cependant, la raison pour laquelle un pays aux chocs symétriques à ceux de la zone devrait être inclus dans celle-ci n'est pas due au faible coût impliqué par cette inclusion, comme chez Mundell, mais plutôt par le coût impliqué par son exclusion.

\section{LA GESTION DES RELATIONS ENTRE L'UEM ET LES DEVISES NON UEM DE L'UE}

Que prédit notre modèle sur la convergence entre pays UEM et non UEM en termes d'inflation qui est une des conditions à l'entrée dans l'UEM ? Selon l'équation (6), une telle convergence ne pourra jamais avoir lieu « naturellement » même si l'Italie réussit à converger en termes réels, c'est-à-dire que $\bar{y}_{\mathrm{I}}$ atteint le niveau $\bar{y}_{F}$ tant qu'elle reste en dehors de la zone de change fixe déjà formée. La création d'une zone de change fixe entre l'Allemagne et la France change en effet les anticipations d'inflation des agents allemands et français mais pas des agents italiens. Si la politique monétaire italienne est décidée librement, l'inflation italienne restera donc supérieure, en moyenne, à celle de ses partenaires. L'exclusion de l'Italie de la zone de change fixe aggrave donc le différentiel de crédibilité et d'inflation qui est à l'origine et qui entretient la volonté d'exclusion de ses partenaires.

Si la convergence monétaire entre l'Italie et les pays de l'UE ne peut pas s'effectuer de manière « naturelle ", un accord de change entre l'UEM et l'Italie pourrait l'y aider. Alexandre Lamfalussy, président de l'IME, a ainsi proposé de « créer un arrangement similaire au mécanisme de change européen de taux de change fixes mais ajustables... " pour stabiliser le taux de change entre la devise européenne et les devises non UEM de l'UE. On peut interpréter cet

1. Financial Times, 13 avril 1995, p. 3. 
équilibre comme décrivant la situation où la France et l'Allemagne forment une UEM et l'Italie fixe son taux de change passivement par rapport à la devise franco-allemande. L'intérêt pour l'Allemagne et la France est évident : un tel arrangement leur permet de bénéficier des gains liés à une zone de change fixe implicite sans avoir à partager la responsabilité de la politique monétaire avec un pays à situation budgétaire difficile. Quel serait alors l'effet de l'imposition d'une relation de type SME entre l'Italie et les pays de l'UEM ? Tout dépend de son impact sur la crédibilité de la politique monétaire italienne. Si les agents croient en l'annonce de l'autorité monétaire italienne qu'elle diminuera l'inflation au niveau de ses partenaires (ou ce qui revient au même dans notre modèle que la lire ne sera pas dépréciée), un système de type SME avec l'UEM permettra ainsi de répliquer les gains de crédibilité d'un système de change fixe pour l'Italie. Cependant, l'expérience récente ne milite pas en faveur d'un tel scénario et on peut craindre des phénomènes d'équilibres multiples où les agents se focalisent sur l'équilibre où l'Italie dévie. En effet, si l'annonce d'un change fixe entre l'Italie et les pays de l'UEM n'est pas crédible, c'est-à-dire que les agents utilisent pour leurs contrats nominaux des anticipations d'inflation élevée, la diminution du taux d'inflation au niveau des autres pays européens serait alors très coûteuse en termes de production.

On peut même penser que la création d'une UEM entre l'Allemagne et la France rendra encore moins crédible pour l'Italie un arrangement de type SME. Supposons que les agents sur les marchés des changes utilisent l'écart d'inflation anticipé entre les monnaies pour calculer leur anticipation de dévaluation en cas de sortie de l'Italie du SME. Cela est tout à fait dans la logique du modèle puisque la PPA tient. Dans le cas d'un SME sans UEM franco-allemande, cela implique que la dévaluation anticipée de la lire en cas de crise du SME est de : $\beta\left(\bar{y}_{\mathrm{I}}-\bar{y}_{\mathrm{A}}\right)$. Si une UEM franco-allemande existe, la dévaluation de la lire en cas de crise du SME est plus forte : $\beta\left(\bar{y}_{I}-0,5 \bar{y}_{A}\right)$ puisque dans ce cas le change fixe franco-allemand est parfaitement crédible impliquant un taux d'inflation francoallemand plus faible en cas d'éclatement du change fixe avec l'Italie. Si ces anticipations de change sont reflétées dans le différentiel de taux d'intérêt via la condition de parité d'intérêt, cela implique qu'un arrangement de type SME sera encore plus coûteux pour l'Italie, et donc moins crédible, en cas d'UEM franco-allemande. En outre, si les agents prennent en compte le gain en termes de stabilisation de la production pour les pays sortant du SME pour calculer la probabilité d'une crise du SME, celle-ci sera plus forte en présence d'une UEM franco-allemande. En effet, sans UEM franco-allemande, l'éclatement du SME ne modifie pas le niveau de la production puisque la production en change fixe ou flexible est de $\varepsilon$. En revanche, en présence d'une UEM allemande, la crise d'un accord de type SME avec l'Italie implique le passage au niveau de production donné par l'équation (7), c'est-à-dire un niveau plus stable pour l'Italie. Le gain potentiel d'une sortie du SME en termes de stabilité de la production pour l'Italie est donc plus élevé avec une UEM que sans UEM. De cet autre point de vue, la crédibilité d'un accord de type SME est diminuée du fait de la création d'une UEM franco-allemande.

Certains, en particulier en France, sont allés plus loin et ont proposé que les membres de l'UEM puissent réagir aux dépréciations compétitives soit en réduisant les montants versés par l'intermédiaire des politiques régionales aux pays qui seraient perçus comme manipulant leur politique monétaire et leur taux de change, soit même en imposant des tarifs sur les exportations de ces pays. 
L'utilisation d'instruments à l'origine de distorsions aussi graves que des tarifs (à la fois pour le pays qui les impose et pour le pays qui les subit), outre qu'elle remet en cause le Marché unique, nous paraît pour le moins dangereuse. De la même manière qu il existe un arbitrage optimal, en équilibre de Nash, entre stabilisation de l'inflation et stabilisation de la production, il existera, dans un tel jeu, un arbitrage optimal entre tarifs et stabilisation de la production pour tous les pays concernés. L'équilibre non coopératif qui en résultera sera donc très sous-optimal du point de vue européen. Il est en outre paradoxal de défendre l'UEM en la présentant comme essentielle pour l'achèvement du marché unique tout en proposant de la mettre à mal directement par l'imposition de tarifs.

L'utilisation des sommes allouées dans le cadre des politiques régionales a au moins l'avantage, à première vue, de ne pas utiliser d'instruments distortionnaires. Elle n'en pose pas moins un certain nombre de problèmes. Supposons d'abord que cette proposition aboutisse à diminuer, de manière linéaire, les transferts vers le pays exclu de l'UEM en fonction du taux de dépréciation d'équilibre de la devise, c'est-à-dire du différentiel d'inflation dans notre modèle. Ceci reviendrait à ajouter dans la fonction de perte de l'Italie un terme tel que : $\gamma\left(\pi_{I}-\pi_{A}\right)$. On peut montrer aisément dans ce cas que l'inflation moyenne à la fois de l'Italie et des pays de l'UEM va diminuer. Le différentiel d'inflation anticipé diminuera donc mais, puisque justement cette diminution est parfaitement anticipée, elle n'aura aucun impact sur les niveaux de production. En effet, la politique monétaire sera toujours aussi contre-cyclique puisque la " punition » n est pas une fonction de l'état de l'économie. En fait, cette proposition rejoint exactement celle faite récemment par Persson et Tabellini [1993] et Walsh [1995]. dans un cadre national, qui proposent que le contrat de la Banque centrale soit une fonction décroissante linéaire du taux d'inflation. Ces auteurs montrent qu' un tel contrat permet de diminuer le biais inflationniste sans affecter la réaction optimale aux chocs. Dans le cadre européen, un tel «contrat » entre par exemple la Commission et les pays hors UEM permettrait donc de réduire le biais inflationniste mais il ne résoudra en rien le problème que nous avons identifié, c'est-à-dire que la variance de l'inflation des pays hors UEM et la variance de la production des pays de l'UEM seront accrues par la politique monétaire du pays exclu ${ }^{1}$. Pour qu'un tel mécanisme ait un impact sur la stabilité de la politique monétaire du pays exclu et donc sur celle de la production des pays de l'UEM, il faudrait qu'il soit une fonction non linéaire du taux de dépréciation de la devise non UEM c'est-à-dire qu'il soit en fait une fonction du choc. La proposition se rapproche alors de celle du «banquier conservateur " de Rogoff [1985] dans un cadre européen. Dans les faits, cela implique que la «punition » (la diminution des transferts) soit plus forte pendant une récession que pendant un boom. On peut aisément anticiper la complexité et les problèmes politiques impliqués par un tel mécanisme. Une autre solution est que l'instrument de réaction soit lui-même procyclique. Il n'est pas certain cependant qu'un tel instrument existe.

1. En revanche, un tel mécanisme permettrait d'éviter le scénario pervers où l'exclusion censée être temporaire devient permanente parce que les exclus ne peuvent jamais converger en termes de taux d'inflation. 


\section{CONCLUSION}

Nous avons analysé quelques problèmes posés par l'exclusion d'un pays de l'UE du processus d'intégration monétaire. L'exclusion a un coût à la fois pour le pays exclu et pour les pays de l'UEM : le pays exclu perd les gains de crédibilité liés aux changes fixes et les pays de l'UEM, en créant une zone de change fixe, permettent au pays exclu d'exploiter le change flexible, ce qui augmente la variance de la production des pays de l'UEM. Nous avons vu que les pays aux chocs les plus symétriques vis-à-vis du pays exclu seront les plus touchés par la politique monétaire du pays exclu. L'exclusion du SME et, à terme, de l'UEM d'un pays de l'UE porte donc en germes des conflits entre les pays de la zone de change fixe et entre ces pays et le pays exclu. L'épisode de la dépréciation de la lire peut donc être mis en perspective dans ce contexte théorique.

La difficulté de la gestion des relations entre les devises UEM et non UEM de l'UE a été, jusqu'à récemment, sous-évaluée. Nous avons montré que les propositions visant à punir les pays hors UEM qui mèneraient des politiques de « dépréciations compétitives » sont, selon leur nature, dangereuses pour le Marché unique, inefficaces ou trop complexes. La solution d'un arrangement de type SME entre les pays exclus et les pays de l'UEM permettrait d'éviter en partie ces problèmes. L'expérience récente du SME prouve cependant que le problème de crédibilité qui a été central dans les crises n'a pas été résolu. Nous avons aussi montré que la formation même d'une UEM partielle accroîtra l'anticipation de dépréciation de la devise exclue et rendra donc un arrangement de type SME encore moins crédible.

\section{RÉFÉRENCES BIBLIOGRAPHIQUES}

Alesina A., Grilli V. [1994], « On the Feasibility of a One OR Multi-speed European Monetary Union », dans Eichengreen B., Frieden J. (eds), The Political Economy of European Monetary Unification, Boulder (Col.) Westview Press, p. 107-128.

ARTUS P. [1995], «L'Union monétaire sera-t-elle attrayante pour les pays périphériques? ", Document de travail, Caisse des dépôts et consignations.

BARRO R., GoRDON D. [1983], « Rules, Discretion and Reputation in a Model of Monetary Policy », Journal of Monetary Economics, 12, p. 101-122.

BAYOUMI T., EICHENGREEN B. [1993], « Shocking Aspects of European-Monetary Unification ", dans Francesco Giavazzi (eds), Adjustment and Growth in the European Monetary Union, Cambridge, Cambridge University Press, p. 193-230.

CEPR [1991], Monitoring European Integration, The Making of Monetary Union.

EICHENGREEN B., GHIRONI F. [1995], « European Monetary Unification : the challenges Ahead ", Discussion Paper, $\mathrm{n}^{\circ}$ 1217, CEPR.

LASKAR D. [1995], «Union monétaire à deux vitesses : Analyse du cour du jeu », Document de travail, CEPREMAP. 
MARTin P. [1994a]. "Monetary Policy and Country Size ", Joumal of Intemational Money and Finance, 13 (5). p. 573-586.

MARTIN P. [1994b], «A Sequential Approach to Regional Integration : Central and Eastem Europe and the EU ", Discussion Paper n ${ }^{\circ} 1070$, CEPR.

MARTIN P. [1995], «Free-Riding, Convergence and Two Speed Monetary Unification in Europe », European Economic Review; 39 (7), p. 1345-1364.

MarTIN P., OTtaVIano G. [1995], «The Geography of Multi-speed Europe », Discussion Paper $\mathrm{n}^{\circ} 1292$, CEPR.

Mundell R. [1961], "A Theory of Optimum Currency Areas », American Economic Review, 51, p. 657-665.

PERSSON T., TABELLIN G. [1993], «Designing Institutions for Monetary Stability », Camegie Rochester Conference Series on Public Policy, 39 (0). décembre, p. 53-84.

RoGofF K. [1985], « The Optimal Degree of Commitment to an Intermediate Monetary Targe ", Quarterly Journal of Economics, 100, novembre, p. 1169-1189.

PISANI-FERRY J. [1995]. "L'Europe à géométrie variable: un essai d'analyse économique ", à paraître dans Politique étrangère.

WALSH C. [1995], "Optimal Contracts for Central Bankers», American Economic Review, 85 (1), p. 150-167. 